\title{
Study on the Intuitive Introduction in Calculus Teaching
}

\author{
Jianxin Dai, Zhixin Li \& Yingfan Liu \\ School of Mathematics and Physics \\ Nanjing University of Posts and Telecommunications \\ Nanjing 210046, China \\ E-mail: daijx js@126.com
}

\begin{abstract}
In the popularization course of higher education, students entering into the higher colleges are not those excellent students with excellent achievements in the senior school, so the teaching of college mathematics in higher colleges should transform from the learning emphasis to popularization emphasis for traditional excellent higher education. In the transformation process, as a main basic course for the students of the engineering course, the calculus has many problems in the teaching. Based on many years' teaching practice and theoretic research, in this article, we first analyze the problems and antinomies faced by calculus teaching, demonstrate the feasibility of intuitive introduction method from the mathematical essential and the rule of human thinking, and finally explain the implementation method of the intuitive introduction combining with concrete teaching cases.
\end{abstract}

Keywords: Higher education popularization, Calculus, Intuitive introduction

Calculus is one of core courses of various specialties for engineering course appointed by State Education Commission of China, and it is one of the most important basic courses, and it not only relates with the learning of various professional courses, but has very important function to cultivate students' cultural and thinking qualities, and every student should study it well. However, the calculus has many characters such as strictness, abstract and system, and it is much higher than the elementary mathematics whether for method or for objective, many students feel it is very difficult. Under the present popularization background of higher education, teachers should emphasize the intuitive of calculus and adopt intuitive introduction to make students deeply understand the concept, theorem, argument and solution, which could cultivate students' study interests, enhance their study efficiencies and enhance the quality of calculus education to some extents.

\section{Problems and antinomies faced by calculus teaching}

(1) The separation of basic education and higher education. Though China middle and primary schools implemented abundant teaching reforms at present, but they could not round the baton of college entrance exam all the while, and the teaching content, mode and requirement of middle and primary schools are not connected with college well, and comparing with the teaching reform of middle and primary schools, the teaching reform of basic college course is lagged, which is adverse for the cultivation of talents.

(2) The college expansion plan brought the problem of uneven student qualities. In recent year, the business of higher education develops very quickly, and the scale of enrollment is continually expanding, and the expansion of freshmen brings the inequality of teaching degree. At present, certain problems exist in some part of students' cognitive ability, quality and study habits.

(3) Single teaching mode. The teaching mode basically adopts the way of "definition + theorem (character, formula) + example (computation)", always emphasizes utilizing formula and reasoning to describe mathematical problems, i.e. "emphasizing the training of signal figure and solution technology, and ignoring the introduction from intuitive aspect and background of problem".

(4) The antinomy between increasing teaching requirements and deficient experienced young teachers. Now many young teachers directly begin to teach after graduation, and though they have higher school experiences and degrees, but they lack certain teaching experiences. Some young teachers thought they are from regular professional training and "grasp" the theory of calculus, but they study few teaching materials and teaching methods, so they are know few to "teach this course well".

(5) Students' dread mentality for mathematical study. Because of improper teaching method and idea, bald teaching content and teaching materials, the deficiency of fascination materials, "over" emphasizing the strictness of mathematical knowledge and the abstract thinking character of mathematical theory and the deficiency of intuitive introduction especial for the geometric intuitive teaching, students lack in study enthusiasm because students have not 
fully realized the support function of mathematics for the development of scientific technology and other subjects, and they thought it is not very important to study the mathematics well.

\section{The feasibility of intuitive introduction in the process of calculus teaching}

The intuitive teaching is to offer abundant cognitions and concrete knowledge and make the formation of concept establish on the base of fact, practicality and idea in the teaching. Some primers in the ancient of China had the character of "the combination of drawing and words". China ancient ideologist Xunkuang said "it is better to hear a thing than not to hear it, still better to see it," "only to know it but not see it, even if erudition is false". In the 17th century, Czech educationalist Johann Amos Comennius alleged that the intuitive teaching is one of golden rules of teaching, "if possible, we should accept everything by our sensibilities", "the dayspring of wisdom is not to learn the items of things, but to really feel the thing itself". Switzerland educationalist J.H. Johann Heinrich Pestalozzi, German educationalist E.A.M. Diesterweg, Russian educationalist Konstantin Ushiksny et al all discussed the intuitive principle and developed the formers' ideas. From the layer of psychology, the intuitive character of teaching is that it is better to see for oneself rather than to hear for many times. In the process of teaching, the implementation of intuitive principle could help to solve many antinomies such as the concept breaks away the thing, the abstract generalization breaks away the concrete image and the understanding breaks away the cognition. Bright and lively image could easily arouse students' attentions, study interests and enthusiasm, promote the understanding and transfer of knowledge, develop students' observation ability, thinking ability and form the opinion of dialectical materialism.

The introduction of mathematicians' work is mathematicians' deep feeling to the mathematics and the mathematical intuition produced from this feeling. In the study of mathematics, we should try to establish the association between abstract the mathematical conclusions and intuitive image. Because the intuitive image is clear and stable in the memory, so it is very easy to remember abstract mathematical conclusions, enhance the memory quality and the study efficiency through it. The intuitive teaching, especially the geometric intuitive teaching should be strengthened. For example, the intuitive teachings of the condition (sufficient and necessary) of function extremum, the definition and application of derivative, the concept and character of convex function, differential coefficient and integral median theorem should be strengthened. Teachers should emphasize the teaching measures and teaching modes, and many intuitive teaching measures such as figure, teaching video, teaching module and computer cartoon could giver intuitive feelings for students in the concept, theorem, agreement and solution, and they are helpful to implement intuitive teaching.

\section{Concrete cases}

(1) Before teachers introduce the geometric meaning of derivative, they can give students such as problem, and as seen in Figure 1, the beeline $\mathrm{AB}$ is the tangent of the curve $y=x^{n}$, ask for the slope of the beeline $\mathrm{AB}$.

First let students drew the figures of $n=2,3,4,5$, then measure the line segments $\mathrm{OC}$ and $\mathrm{AC}$, compute the proportion, and finally obtain the conclusion $\frac{|O C|}{|A C|}=n$, but $|O C|=x,|B C|=y$, so the slope $k=\frac{|B C|}{|A C|}=\frac{x^{n}}{\frac{x}{n}}=n x^{n-1}$. It is just the derivative of the power function $y=x^{n}$. On the one hand, we can get the derivation of the power function by the method of primary mathematics, and on the other hand, the real process of intuitively simulating the mathematical discovering could deepen students' understanding for the geometric meaning of derivative and make students enjoy the pleasure of mathematical discovering.

(2) We can use the normal method to beg the integral $\int_{0}^{1}\left(\sqrt{1-x^{2}}-x+1\right) d x$, but it is more simple from the geometric meaning of definite integral. As seen in Figure 2, the integral could be taken as the area of the plane figure surrounded by curves $x=0, x^{2}+y^{2}=1, y=x-1$, so it is easy to know the area is $\frac{\pi}{4}+\frac{1}{2}$. This method is very intuitive, and it could deepen students' understanding for the definite integral and inspire students' study enthusiasm for mathematics, and establish strong base for the successive course.

Another example is to compute the dual integral $\iint_{D}(5 x+3 y) \mathrm{d} x \mathrm{~d} y$, where $\mathrm{D}$ is the plane field surrounded by the curve $x^{2}+y^{2}+2 x-4 y-4=0$.

As seen in Figure 3, the integral area is $(x+1)^{2}+(y-2)^{2} \leq 3^{2}$, and its centroid coordinates is $\bar{x}=-1, \bar{y}=2$, the area is $A=9 \pi$, and the formulas of centroid coordinates are $\bar{x}=\frac{1}{A} \iint_{D} x \mathrm{~d} x \mathrm{~d} y$ and $\bar{y}=\frac{1}{A} \iint_{D} y \mathrm{~d} x \mathrm{~d} y$, so it is very easy to solve the integral by the centroid coordinates combining the integral area. 


$$
\text { formula }=5 \iint_{D} x \mathrm{~d} x \mathrm{~d} y+3 \iint_{D} y \mathrm{~d} x \mathrm{~d} y=5 \cdot \bar{x} A+3 \cdot \bar{y} A=[5 \cdot(-1)+3 \cdot 2] A=9 \pi
$$

(3) About the conclusion that the partial derivative of multivariate function can not be ensured to be consecutive, though the counterexample is in the book, but some students are difficult to understand it, and they still thought the derivative is necessarily consecutive, so here the intuitive analysis is very effective. Taking the dual function as the example, the partial derivative of $z=f(x, y)$ exists at the point of $\left(x_{0}, y_{0}\right)$, i.e. $\lim _{x \rightarrow x_{0}} \frac{f\left(x, y_{0}\right)-f\left(x_{0}, y_{0}\right)}{x-x_{0}}$ and $\lim _{y \rightarrow y_{0}} \frac{f\left(x_{0}, y\right)-f\left(x_{0}, y_{0}\right)}{y-y_{0}}$ exist, and here the variable range of the variables we should consider respectively are two intervals seen in Figure 4.

$z=f(x, y)$ is consecutive at the point of $\left(x_{0}, y_{0}\right)$, i.e. $\lim _{\substack{x \rightarrow x_{0} \\ y \rightarrow y_{0}}} f(x, y)=f\left(x_{0}, y_{0}\right)=A$, and here the variable range we should consider is the circular field seen in Figure 5.

When it is consecutive, the change range of variable is much than the derivative range, so it is obvious that the partial derivative exists and it can not be ensured to be consecutive.

In a word, if we emphasize the intuitive introduction in daily teaching process and teachers could discuss and communicate their experiences, the quality of calculus teaching could be enhanced largely.

\section{References}

Felix Klein, interpreted by Zhu, Xuexian. (2002). Ancient and Modern Mathematical Ideas. Shanghai: Shanghai Science Technology Press.

Yang, Limin. (2003). Regarding Intuitionist Way in Higher Mathematic Teaching. College Mathematics. No.19(2). P.34-37.

Yang, Xiaoping \& Xu, Chungen. (2006). Strengthening Intuitive and Application Teaching and Enhancing the Educational Quality of College Mathematics. College Mathematics. No.22(6). P.1-3.

Yu, Guangyuan \& Wang, Wenqin. (2003). Discussion on the Intuitive Principle in the Process of College Teaching. Journal of Hexi University. No.19(6). P.103-106.

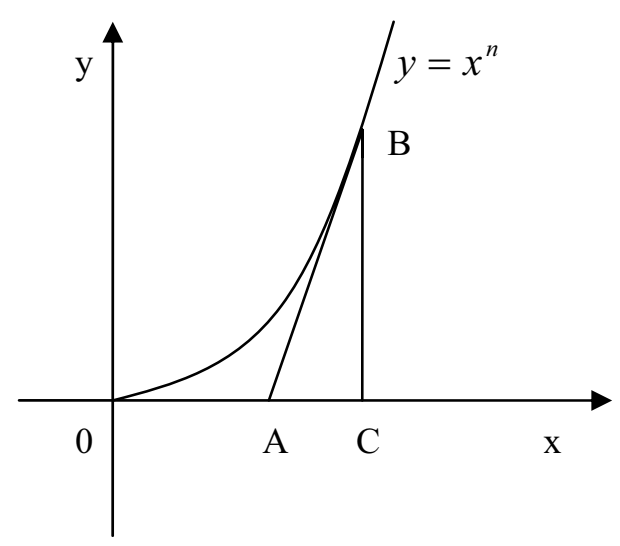

Figure 1 


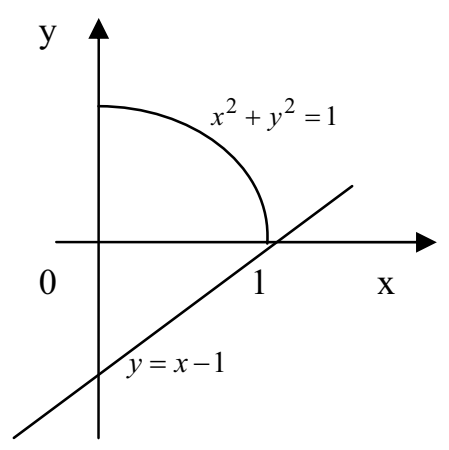

Figure 2

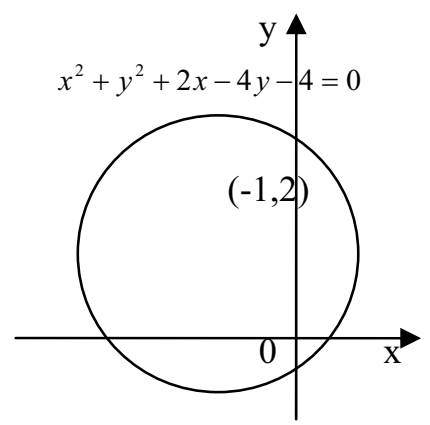

Figure 3

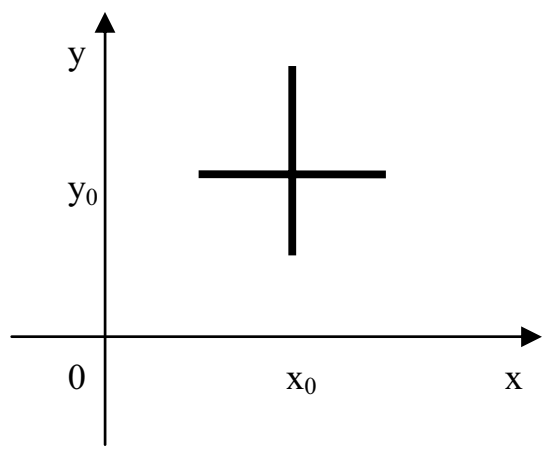

Figure 4

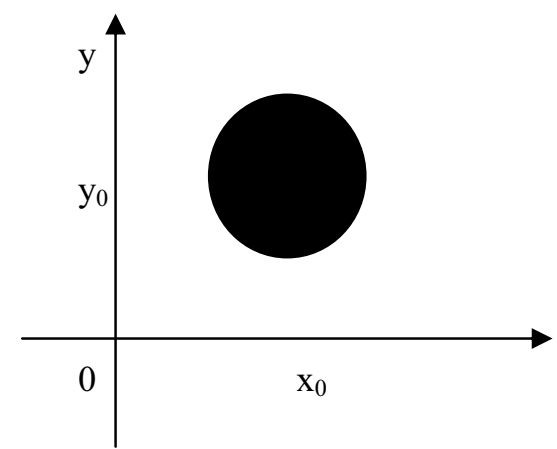

Figure 5 